\title{
A decision analytic model to guide early-stage government regulatory action: Applications for synthetic biology
}

\author{
Benjamin Trump \\ Department of Health Management and Policy, School of Public Health, University of Michigan, Ann Arbor, MI, USA \\ Christopher Cummings \\ Wee Kim Wee School of Communication and Information, Nanyang Technological University, Singapore \\ Jennifer Kuzma \\ School of Public and International Affairs and Genetic Engineering and Society Center, North Carolina State University, Raleigh, NC, USA \\ Igor Linkov \\ US Army Engineer Research and Development Center, Concord, MA, USA
}

\begin{abstract}
Synthetic biology (SB) involves the alteration of living cells and biomolecules for specific purposes. Products developed using these approaches could have significant societal benefits, but also pose uncertain risks to human and environmental health. Policymakers currently face decisions regarding how stringently to regulate and monitor various SB applications. This is a complex task, in which policymakers must balance uncertain economic, political, social, and health-related decision factors associated with SB use. We argue that formal decision analytical tools could serve as a method to integrate available evidence-based information and expert judgment on the impacts associated with SB innovations, synthesize that information into quantitative indicators, and serve as the first step toward guiding governance of these emerging technologies. For this paper, we apply multi-criteria decision analysis to a specific case of SB, a micro-robot based on biological cells called "cyberplasm." We use data from a Delphi study to assess cyberplasm governance options and demonstrate how such decision tools may be used for assessments of SB oversight.
\end{abstract}

Keywords: emerging technology, risk governance, synthetic biology, technology governance, uncertainty.

\section{Introduction}

Synthetic biology (SB) seeks to apply the principles of biological systems engineering through the design and assembly of biologically inspired parts and systems that do not exist in nature in order to rewrite natural biological systems for useful purposes. SB comes in many forms, from the genetic engineering of metabolic pathways into existing organisms to the bottom-up construction of biological systems from macromolecule components to living systems based on amino acids or genetic material that are synthesized (deLorenzo 2010). A key example of SB was demonstrated by the J Craig Venter Institute in 2010 when they developed a synthetic genome for the bacterium Mycoplasma mycoides and implanted it within a bacterial shell that had its own genome removed (Gibson et al. 2010; Hutchison et al. 2016). Since then, visions of SB propose benefits to society across multiple sectors and improvement in a variety of processes, ranging from environmental remediation to vaccine development to biofuel production, among others. However, SB is also expected to be controversial for many reasons, including value-based objections of human interactions with nature to concerns about potential human health, environmental, and socioeconomic risks (Kuzma \& Tanji 2010; Church et al. 2014).

Correspondence: Benjamin Trump, Department of Health Management and Policy, School of Public Health, University of Michigan, 1415 Washington Heights, Ann Arbor, MI 48109, USA. Email: bdt2011@gmail.com

Accepted for publication 13 October 2016. 
For example, one SB application currently under development, "cyberplasm" converges synthetic biology, biomimicry, nanotechnology, and robotics to construct a micro-scale robot designed to be capable of sensing environmental chemicals for remediating purposes (Voigt 2012). It has been proposed for use in detecting and treating pathogens within plants and animals in situ. Cyberplasm is a biohybrid that integrates engineered bacteria, yeast, and mammalian cells to undertake device-like functions modeled after the sea lamprey. It is expected to be in full development with a functional prototype by 2017.

As engineered microorganisms such as cyberplasm are envisioned to be biohybrids, it is likely that such technologies will "belong in a different risk category than toxic chemicals or radioactive materials" and will not fit risk assessment approaches for living, genetically engineered, or other organisms where risks associated with gene flow and invasiveness are of concern (Tucker \& Zilinskas 2006, 31). Appropriate governance mechanisms have the potential to diminish chances of serious harm and maximize the likelihood that benefits of SB can be shared across a broad spectrum of society while respecting a diverse array of societal values. Schmidt et al. contend that SB requires "new methods of risk assessment to decide whether a new SB technique [...] is safe enough (for human health, animals and the environment) for the use in restricted and/or less restricted environments" (2009, p.178). Such distinction from established domains of risk characterization, assessment, and governance make any traditional risk assessment potentially irrelevant to SB products because of the potentially unique risks and uncertainties posed by the use of selfreplicating microorganisms. While many effective governance structures for managing technological developments are currently in place around the world, there may be a need to evolve and forecast governance actions because of the revolutionary nature of SB technologies like cyberplasm. As SB requires potentially new and unique measures to govern the risks associated with human and environmental health, policymakers for national governments are already being forced to make decisions regarding how stringently to regulate, litigate, and monitor the various emerging technologies that are in development or coming to market (Mohan et al. 2012).

This is a complex task because of the inclusion of various stakeholders amidst a dearth of objective information. Further complicating matters are the various factors that must be considered with any policy decision in this regard, where policymakers are required to balance economic, political, social, and scientific risks and benefits associated with the use of such technology. Such high-stakes decisions must often be undertaken in the absence of robust quantitative data or extensive field research, making it more difficult for policymakers to identify optimal response options for governing SB applications as they develop. To better understand these issues, some have suggested that researchers make use of social scientific methods to demystify potential risk management strategies that could be employed given the technology's early stage development and use (Calvert \& Martin 2009). Others have categorized SB products according to sector and technologies involved (e.g. kinds of parts or methods) to identify risk categories of most concern in order to develop scales of "precaution to promotion" that may be warranted (Kuzma \& Tanji 2010).

Risk governance for emerging technologies has been given special attention in the literature because of the lack of knowledge and experience with potential hazards, their interactions with systems in which they are embedded, and their impacts. Emerging risks are characterized by uncertainty about potential consequences and probabilities of occurrence. The International Risk Governance Council (IRGC) has recommended that when risk problems involve high uncertainty and ambiguity, processes limited to traditional agency and expert-driven risk analysis based on quantitative data will not suffice (International Risk Governance Council [IRGC] 2006, 2015). If there is a high degree of uncertainty, hazard criteria of reversibility, persistence, and ubiquity should be considered. Also, risk management should include more cautionary approaches, such as close monitoring, small steps in introducing the hazard, strict containment, and systems that can absorb or tolerate surprises and that are resilient, flexible, and adaptive (IRGC 2006). In situations of high ambiguity, there is no agreement among stakeholders and experts as to the questions of most importance for analyzing or mitigating risk, and there are conflicting values about what is to be protected or what risks should be reduced. Under high ambiguity and uncertainty, broader societal discourse is most appropriate to frame problems and management options with participation from a wide range of stakeholders and citizens (IRGC 2006).

In this paper, we propose a decision tool to aid policymakers, stakeholders, and societies with the task of assessing governance options for various applications of SB innovation. While such applications of SB may or may not come to commercial fruition, this decision aid affords regulators, policymakers, and stakeholders the opportunity to assess various tools of governance at their disposal for a variety of SB applications. This decision aid may also help to make appropriate policy judgments based upon qualitative expert-derived information in lieu of unavailable quantitative experimental data. This tool can be integrated into wider deliberative processes suggested 
by the IRGC to help stakeholders and experts develop scenarios and frame management options together (IRGC 2015). In this way, such a tool systematically quantifies and makes use of expert and stakeholder opinion that may not always be reflected in the traditional regulatory policymaking process. We sought to identify answers to the following research questions:

1 What governance options could be proposed regarding the potential applications of cyberplasm?

2 What is a good process for distilling expert derived data to recommend policy options for SB products, such as with cyberplasm?

Answering both of these questions requires methodological approaches to: (i) acquire and interpret information pertinent to the risks and benefits of cyberplasm, and (ii) analyze and rank decision alternatives based upon these risks and benefits. To accomplish these aims, we make collective use of the Delphi methods to generate qualitative expert interviews, as well as employing multi-criteria decision analysis (MCDA) to assess value judgments and rank governance alternatives for cyberplasm based upon expert input and discussion within scholarly literature (Linkov et al. 2013; Bates et al. 2016). Specifically, Question 1 is addressed through the use of the Delphi method, where individual and collective opinion is assessed from subject experts to identify cyberplasm governance in the technology's early stage of development (Linstone \& Turoff 2002; Berube et al. 2011). Question 2 is addressed through the use of MCDA, which integrates qualitative findings from subject experts into a decision model that ranks governance options by their degree of perceived optimality (Linkov et al. 2013; Bates et al. 2016).

When applications of emerging technologies, such as with SB, are generally lacking substantial objective data, the use of subject expert opinions regarding the potential risks and benefits is required in order to inform policy decisions related to how the technology should be regulated and governed. Transparent decision analytical tools such as MCDA make this task possible in the immediate term. Using MCDA, expert opinion and judgment may be aggregated in a formal and quantitative manner, ultimately affording its user the potential to make value judgments and trade-offs based upon the perceived risks and benefits of the technology. Collectively, the use of the Delphi method (Linstone \& Turoff, 2002; Berube et al. 2011) and MCDA (Linkov et al. 2013; Bates et al. 2016) have been discussed as approaches that can inform governance of emerging technologies within an environment of high uncertainty. Such an approach may be helpful for regulators and other stakeholders seeking to drive the risk governance of synthetic biology products, and this paper demonstrates how this process would work for the case of cyberplasm governance.

\section{Case study}

Modeled after the sea lamprey, cyberplasm is a biohybrid that integrates engineered mammalian cells and sensory neurons to undertake device-like functions (Voigt 2012). The sea lamprey serves as the biological model for the cyberplasm's design. The animal swims by undulating its long tail in an uncomplicated wavelike motion and it also has a relatively primitive nervous system. These characteristics are easy to mimic in terms of modeling. Biomimetic, synthetic muscles are being designed to initiate the undulatory movement that will propel the micro-robot through water, while sensors derived from yeast cells report environmental signals (Grubišić \& Parpura 2015). Such signals allow the micro-robot to move toward their electronic stimulus to collect environmental data or deliver medical treatment on site. Such a framework requires a strong integration of biological sensory neurons with the artificial electrical ones, involving a level of interface scaffolding that facilitates the relay of signals throughout the nervous system. This is accomplished through the use of ultraviolet light on a silicon-derived organic polymer with a water coating. Through the additional integration of carbon nanotubes, the scaffold is constructed to be electrically conductive and react to predetermined stimuli (Szondy 2012).

Researchers have speculated that in the long-term, cyberplasm could eventually impact the healthcare industry by improving biosensors and drug delivery systems through cellular machines after injection into the human body. Cyberplasm is currently being researched and developed by an international team of scientists and engineers in the United States (US) and the United Kingdom (UK), jointly funded by the National Science Foundation and the Physical Sciences Research Council, respectively (Woodrow Wilson Center 2016). Oversight and risk analysis for such an SB project is likely to be difficult given the convergent nature of the technologies used and the broad possibilities for deployment. 


\section{Methods}

This study employs MCDA to data acquired from a Policy Delphi study to assess cyberplasm governance (Linstone \& Turoff 2002). Methodologically, the use of the multi-round Delphi study allows for a consideration of individual and collective discussion by identified subject experts on the subject of cyberplasm governance. This approach utilizes the strengths of such a qualitative approach by acquiring a context-rich assessment of an SB product whose risks and benefits would currently be impossible to quantitatively assess, while also mitigating the Delphi method's traditional weaknesses by acquiring individual opinions and insight into the technology's risks prior to group discussion (Linstone \& Turoff 2002). Likewise, MCDA serves as a method to utilize information derived from subject expert interviews and rank decision alternatives via critical factors of consideration known as decision criteria (Bates et al. 2016). MCDA may be of particular use to review governance options for emerging technologies like SB - the method offers some insight via expert elicitation regarding value trade-offs and uncertainties that $\mathrm{SB}$ products may possess in their early stages of development (Linkov et al. 2013; Bates et al. 2016; Subramanian et al. 2016).

The purposive sample of experts was chosen to provide "information rich" cases that can provide greater depth of insight into the potential policy impacts and issues of cyberplasm governance. Individuals in various professional disciplines are deliberately included to gain multiple perspectives on the technology and its proper governance (Patton 2005). The list of potential panel members was compiled using a mixture of web database searches, literature reviews for relevant author names, and prior knowledge and experience of the research team. The initial listing contained 234 experts originating from a variety of groups, including: attendance lists from research conferences and national and international professional association meetings; editorial review boards for SB research journals; and government listed panels of research, policy, and ethics review committees. Additional potential panel members were also added to the list based on personal review from other SB researchers and analysts, as well as the research team.

The initial panel members represented a wide range of disciplines $(n=48)$ including chemistry, molecular biology, and bio-engineering ( $n=14$, including those working on biological parts such as those used in cyberplasm who gave firsthand accounts of risk and regulatory needs of the technology); policy, governance, and law ( $n=14)$; ecology, environmental science, and toxicology $(n=6)$; and bioethics, philosophy, sociology, and science and technology studies (STS) $(n=14)$. Thirty-five members completed the four rounds of the Delphi study (Table 1). Potential participation contacts were limited because of the novelty of SB, as active research and development are limited to only a few dozen well-known scholars and developers active in publication and conference presentations; such a respondent count is consistent with similar research efforts on SB governance (Bates et al. 2016).

This Delphi study elicited expert judgments about the potential benefits and risks of cyberplasm, as well as judgments regarding the ethical, legal, and societal issues of the technology. Named after the oracle of Delphi, the Delphi method was designed to forecast decisionmaking under uncertain conditions when accurate information is unavailable for ethical or financial reasons, or when subjective information is all that is available for a decision or policy assessment. In a typical Delphi study, expert panel members respond to sets of open-ended and closed-ended questions individually, anonymously, and at their leisure, thus reducing group effect biases and allowing panel members to voice and

Table 1 Delphi rounds and participants

\begin{tabular}{|c|c|c|}
\hline Round & Method & Description \\
\hline One & $\begin{array}{l}\text { Standardized open-ended interview } \\
\text { protocol }(n=45)\end{array}$ & $\begin{array}{l}\text { Participants responded to a variety of questions about risk analysis, governance, } \\
\text { and societal issues for cyberplasm, as well as the other three cases (biomining, de- } \\
\text { extinction, and plant microbes for nitrogen-fixation). Interviews were } \\
\text { approximately } 75 \text { minutes in length. }\end{array}$ \\
\hline Two & Online quantitative survey $(n=34)$ & $\begin{array}{l}\text { Designed from preliminary round one findings. Included various scaled items } \\
\text { regarding risk governance issues associated with the case studies. }\end{array}$ \\
\hline Three & $\begin{array}{l}\text { Face-to-face workshop and ordinal } \\
\text { ranking exercise }(n=35)\end{array}$ & $\begin{array}{l}\text { Focused on concept-mapping and mind-mapping exercises to generate lists of } \\
\text { challenges and opportunities for SB governance. Also included an ordinal } \\
\text { ranking exercise of ideal governance characteristics. }\end{array}$ \\
\hline Four & $\begin{array}{l}\text { Online qualitative and quantitative } \\
\text { survey }(n=35)\end{array}$ & $\begin{array}{l}\text { Open-ended and closed-ended items and scales assessing factors that may } \\
\text { influence future policy and governance options concerning the case studies. }\end{array}$ \\
\hline
\end{tabular}

$\mathrm{SB}$, synthetic biology. 
change their opinions without fear of repercussion. This method also encourages the equal flow of information between panel members and researchers and grants adequate time for experts to reflect on developing issues within the study itself. This project used a modified version of the Delphi study, a Policy Delphi study, where the primary goal is to "have an informed group present all the options and supporting evidence," regarding the case of cyberplasm (Linstone \& Turoff 2002, p. 80). Given the uncertainties related to the risks and benefits posed by cyberplasm, a Policy Delphi study was chosen in order to generate individual context-rich insight into the technology across the included experts while also promoting group discussion and debate afterwards to discuss concerns or uncertainties related to the technology's characteristics and development (Berube et al. 2011; Linstone \& Turoff 2002). Data presented forthwith in this study were obtained from various phases throughout the Delphi study and the results are derived solely from qualitative and quantitative data collected during the project.

Table 1 contains information regarding the project's four-round elicitation process, including the number of respondents at each stage, the type of elicitation conducted, and other general information.

\section{Multi-criteria decision analysis model}

To integrate and analyze responses from Delphi participants, we utilized MCDA, which is a method of decision analysis that facilitates value trade-offs of complex and uncertain issues with various sources of information (Linkov \& Moberg 2011). Specifically, MCDA integrates qualitative and quantitative information alike, allowing experts to voice their beliefs and opinions on a subject with limited field data or formal scientific agreement (Linkov \& Moberg 2011; Linkov et al. 2013). Further, MCDA tools can be used to review any disagreements or uncertainties signaled by subject experts through the use of sensitivity analysis, making MCDA all the more helpful for regulators that have to address shifting political, economic, social, and environmental factors as more information about specific products becomes available (Saltelli et al. 1999; Linkov et al. 2013). Given these factors, MCDA serves as an appropriate method to analyze information derived from the Delphi process and rank governance options for cyberplasm based upon relevant decision criteria.

For this particular case, we make use of DECERNS decision analytic software, which allows for a multi-method comparison of several decision analytical tools for a single dataset (Yatsalo et al. 2015). Other MCDA models have been developed to assess the performance of oversight from a retrospective perspective, and have also been applied to case studies, such as with genetically engineered organisms (Kuzma et al. 2008, 2009). This study also uses the case of technological product and multiple criteria for evaluation, but takes a prospective approach to help with future decisions about emerging technologies.

Specifically, we utilized multi-attribute value theory (MAVT), in which expert perceptions of the technology's characteristics are converted to values and evaluated alongside subjective weights and information. This may be represented mathematically as: $V(a)=F(V 1(a 1), \ldots, V m a m))$. The overall objective of this methodology is to represent the decisionmaker's opinions and preferences in the value function $\mathrm{v}(\mathrm{a})$, where $a$ symbolizes the vector of criteria to be evaluated, $w m$ the weighted values, and $V i(\mathrm{x})$ the partial value function for the criterion $\mathrm{Ci}$ (Yatsalo et al. 2007). MAVT maintains underlying assumptions of stakeholder rationality and consistency in judgment, which we sought to assess throughout the interview process (Belton 1986). We illustrate the process of our model's creation in Figure 1, and further explain how decision criteria and alternatives were constructed and scored.

The first step behind the construction of our value hierarchy consisted of identifying the various criteria by which a decisionmaker would evaluate the risks and benefits of a cyberplasm product. This effort was driven primarily by assessment of qualitative interview transcripts and responses from rounds 1 and 2. For additional argumentative validation behind a criteria's construction, suggestions from SB risk governance literature were reviewed for consistency with expert interview responses. This literature was constrained to articles that discussed the implications as well as the governance of SB, including articles that discussed the prospective risks and benefits that SB products (particularly cyberplasm) may offer to our decision criteria. With both sets of available information, the authors focused criteria construction on those elements that were mentioned by more than one expert, and were present for discussion as relevant in the consideration of risk and benefit by at least two articles published in peer-reviewed journals. This approach is similar to the one used by Bates et al. (2016) and Linkov et al. (2012), who utilized literature and expert insight alike to identify important factors for consideration that would influence MCDA output. Definitions for these criteria were 
Criteria Hierarchy

Formation

R1-2 Interview Suggestions \&

Analysis of Scholarly Literature

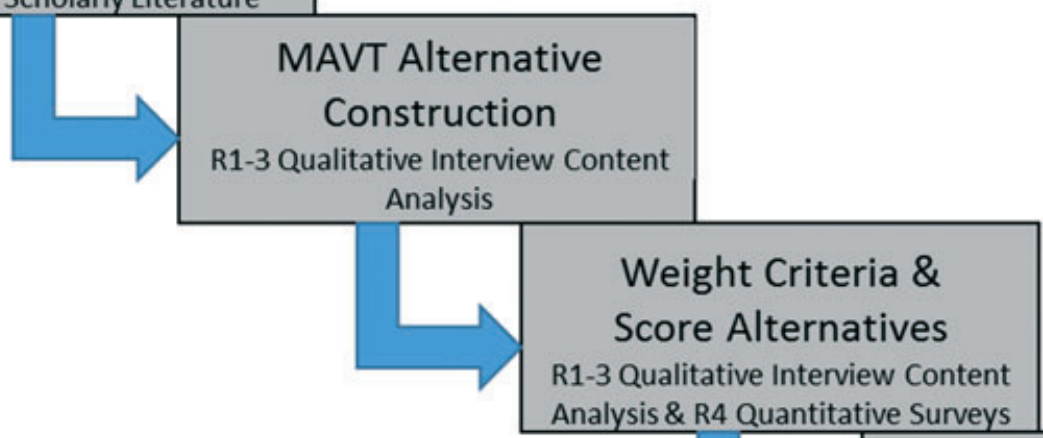

quantitative survey responses from four rounds of subject expert interviews.

derived in a rank-maximizing fashion to make results found later in MAVT calculation more intuitive to readers regarding cyberplasm risk and benefit.

Using information generated from expert interviews and confirmed by emerging literature, the second step included assessing the trade-offs and optimality of six alternative sets of policies that could be pursued by a given nation's regulatory bodies. Similar to criteria and sub-criteria formation in step one, alternatives construction was derived by reviewing comments made in interview transcripts in interview rounds 1 and 2 alongside governance responses from round 3. Similarly, additional justification was derived from scholarly literature regarding the possibility that such a governance alternative could be deployed by a government agency for SB products in general. While these options are simplified because of the emerging nature of the field, they do cover a wide breadth of potential regulatory options, and could be easily modified in the future as SB applications for cyberplasm mature and commercialize. These options are arranged in order of restriction placed upon an individual researcher or scientist in the field, including:

1 No Governance - no new or specific regulatory actions taken to govern or restrict SB research and development.

2 Internal review board (IRB) pre-research - research requires novel or technology-specific checkpoints, which are monitored by within-organization review boards.

3 External review board (ERB) post-research - research requires debrief with external government agency after completion or at certain checkpoints.

4 ERB pre-research - research proposals require approval and oversight from an external government review board prior to research being conducted.

5 Secure labs - research is restricted to government-monitored laboratories only.

6 Moratorium - additional research and development is banned.Together, placeholders constructed for the decision criteria for cyberplasm governance and policy alternatives enabled us to build the model's value hierarchy, or the general structure showing relationships between decision criteria and policy alternatives. In Figure 2, we detail the value hierarchy that breaks down the criteria and alternatives that must be considered in cyberplasm's multi-criteria decision framework.

After constructing the hierarchy, we reviewed interview transcripts and surveys with subject experts to acquire weights for each criterion (in yellow), and scores for each subcriteria alternative linkage (in gray). Criteria weights were derived from responses in interview rounds 2 and 4, while alternative scores were derived from survey responses and transcript comments from rounds 2 and 3. Further, criteria weights were validated by question-specific ordinal scale 


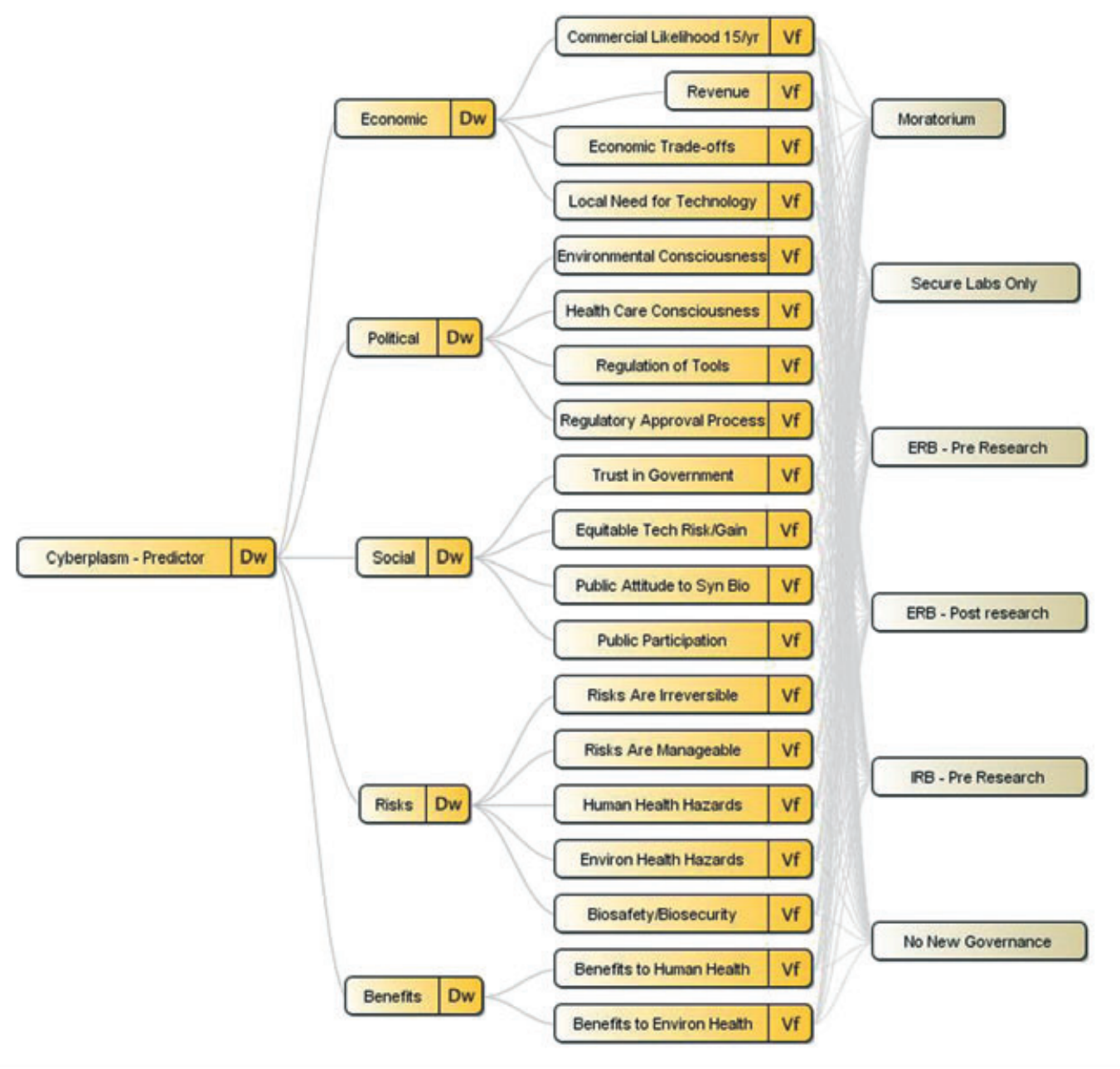

Figure 2 Value hierarchy of cyberplasm governance and decisionmaking via product risk and benefit. ERB, IRB, Syn Bio

survey questions, allowing for an indication of the relative importance that a criterion should have based upon existing knowledge of SB governance and decisionmaking. Likewise, alternative scores were derived by a mixture of questionspecific ordinal scale survey questions where available, or through indications of strength of statement made by respondents alongside the relative confidence expressed by respondents through qualitative interview transcripts and responses in discussing a particular risk or benefit related to cyberplasm governance options. In cases where respondents did not express confidence in their answers, or discussion for a particular policy alternative was limited, further consideration of SB governance literature related to efforts to mitigate risk and promote benefit was included in alternative scoring. This was minimized as much as possible, although general literature perusal was conducted for all decision alternatives to promote consistency in the methodological approach for our model.

With regard to criteria weighting, survey questions asked specific queries about particular model criteria on a scale of 1-10, where each response signifies the general level of importance that a particular criteria factors into an overall SB policy decision. Individual criteria values were assessed as a simple arithmetic mean (see Eqn 1). Any blank responses caused that particular respondent to be removed from average calculations for that given criteria. Later, all criteria values sharing a root source were scaled using unity-based normalization (also in Eqn 2).

$$
\begin{aligned}
& a=\frac{1}{n} \sum_{i=1}^{n} x_{i} \\
& \mathrm{x}^{\prime}=\frac{a-\min (x)}{\operatorname{Max}(x)-\operatorname{Min}(x)}
\end{aligned}
$$


Specific to alternative scoring, such quotes sought in transcript information included: "the environmental health impacts are not at all important in this case," which contributed to a score of 0 to most alternatives' environmental health score; or statements such as "I think that kind of less formal structures of governance that are less oriented around regulations but more thinking about the shaping of technologies [...] is actually something really important" generally favored alternative scores with less restrictive governance overall. As a coding rule, each scoring item required a minimum of two direct quotes to gauge the score, with separate coding passes taken and discrepancies reviewed by the investigators. Quantitatively, alternative scores were assessed on a rank-maximized integer scale ranging from 0 to 3 , where each integer may be understood as: 0 , no improvement; 1 , minor potential improvement; 2 , significant potential improvement; and 3, strong inevitable improvement.

Two reviewers were instructed to review specific key phrases within interview text and score alternatives accordingly. Such key phrases centered on the degree of importance placed by the respondent upon the risk factors and regulatory needs of cyberplasm in a given context (i.e. economic or environmental). Further, the judges were told to base their scores on at least four specific comments within interview text, and were to contrast these phrases with any differing opinions noted by other experts. The two score sheets were compared for consistency, and inconsistent scores were reviewed by both judges to arrive at a consistent result. It is important to note that no inconsistency between the two reviewers was greater than 1 integer, indicating that no significant gap in respondent perception arose.

\section{Results}

Model calculation involved the aggregation of score and criteria weights, and a subsequent summation of these products across all criteria (or weighted sum). The resultant final score serves as an evaluation of each policy alternative's optimality, with a higher score being more favorable. This standard model was evaluated alongside a model detailing the level of confidence applied by each subject to their answer, or the level of surety that their answer was the correct one. This comparison allowed for a review of whether "wild guesses" skewed the model significantly, or biased the results of a particular policy alternative.

For each of the six policy alternatives presented here, a net score was calculated, as shown in Figure 3. These calculations indicated small net performance optimality for the ERB pre-research alternative (score of 0.76). However, the secure labs only (0.64), IRB pre-research (0.61), and ERB post-research (0.59) alternatives also performed well. We will further discuss quantitative reasons why the alternatives score as they do, and perform sensitivity analysis to determine which conditions the existing optimality may shift (Saltelli et al. 1999). Such an assessment allows for an understanding of how resilient and dependable the selection of ERB pre-research is as the highest scoring governance option for SB cyberplasm research and development (Saltelli et al. 1999; Stewart 2005).

To gain an additional understanding of the uncertainty and relative strength of expert knowledge, we review the confidence scores (or the confidence expressed by subject experts related to the perceived risks and benefits that a cyberplasm product poses for a given criteria) for the final raw results alongside the collective aggregation of the five major criteria and their relative subcriteria. In Figure 4, the results of this analysis demonstrate general overall consistency in the ranked list of results ( \pm 2.5 percent or less in each of the six policy alternatives). This indicates that despite some uncertainty in respondent answers, responses do not generally show any major outliers or points of concern that could shift optimality in the final results. Unpacking this by reviewing the confidence-weighted scores for the individual criteria weights is more distorted, particularly for the social criteria weight, which maintained an overall lower level of confidence relative to the other criteria weights. Regardless, all but one individual data point falls below the 60 percent level of confidence, or roughly a \pm 5 percent difference from the overall confidence-weighted final score.

Lastly, we sought to further test the magnitude of the difference in scores by conducting sensitivity analysis on the primary criteria's weights (an example of the economic criteria is shown in Figure 5) (Saltelli et al. 1999; Linkov et al. 2012 , 2013). Specifically, we reviewed the impact of increasing or reducing the degree of importance assigned to a given criterion, with the difference removed or added to the other five criteria distributed equally.

Our sensitivity analysis indicates at least one crossover point in the weight distribution of each criteria, where the different alternative may achieve a top score based upon a shift in criteria weights. However, not all of these crossover points are reasonable, such as if social were given a weight approaching 0 (meaning that stakeholders place virtually no value on social criteria to SB policy and decisionmaking) or if benefits were assigned a weight approaching 1 (meaning that stakeholders only care about benefits as opposed to various concerns for other criteria). While optimality may shift 


\section{Report (MAVT)}



\section{Resulting values of alternatives}

\section{IRB - Pre-research score $=0.611$ \\ Moratorium score $=0.527$ \\ No New Governance score $=0.392$ \\ Secure Labs Only score $=0.640$}

Figure 3 Cyberplasm governance alternatives: ranked list. ERB, IRB, MAVT,

or reach a point of ambivalence at the ends of the spectrum for these criteria, these scenarios are highly unlikely and are generally unrealistic.

More realistically, the other three criteria (risks, political, economic) each contain possible crossover points that, if weights on a given criterion moved from one side of the breakeven point to another, the model would call for a different approach to cyberplasm governance. Specifically, the top scoring option switches from ERB pre-research to secure labs and eventually even full moratorium once the criteria weight for risk reaches 64 percent (assuming all other criteria

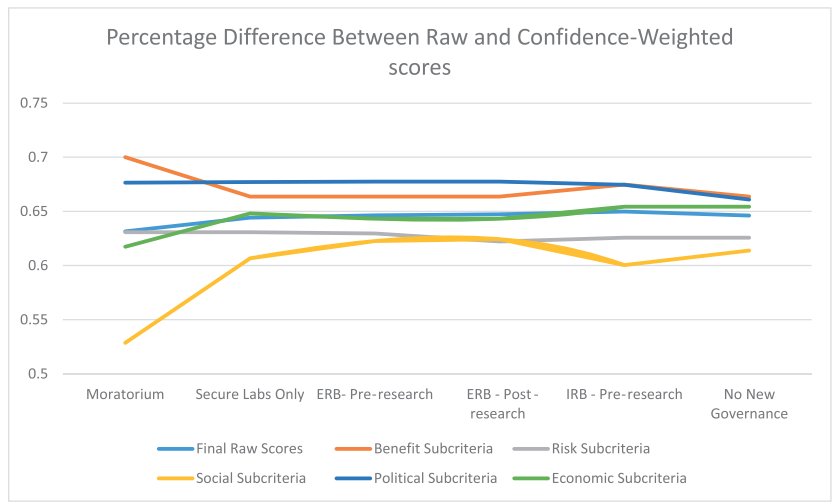

Figure 4 Percentage differences between raw and confidence-weighted scores. ERB, IRB, 




Figure 5 Sensitivity output: economic criteria weighting shift. ERB, IRB,

weights are reduced proportionally). While this may seem distant from the current weight of 23.7 percent, it is within the realm of possibility that, should a strong paradigm shift take place where government officials and stakeholders place significantly more weight on avoiding the risk of emerging technologies, the optimal policy alternative to cyberplasm development should be increasingly restricted commensurate with the government's appetite for hazard.

The remaining two criteria (economic and political) also contain crossover points that generate a sensible narrative based upon the driving factors that each criteria covers. For instance, the more a government decisionmaker cares for the economic criteria driving cyberplasm development, the less stringent the optimal regulatory policy alternative would become (from ERB pre-research to IRB pre-research to no new governance). Likewise, for the political criteria, the more that decisionmakers value political factors behind emerging technology, the more likely they are to restrict such technology development from taking place because of the potential negative outcomes that could accrue. While these individual inflection points may or may not be interesting and realistic in their own right, they do represent potential concern that, sometime in the future, changing priorities among government stakeholders and the public may shift in such a way as to dramatically impact decisionmaking in this field. Although any shift in the optimal governance alternative would require a substantial shift in priorities and risk/benefit trade-offs by regulatory decisionmakers, the potential for such optimality to shift does warrant some discussion into the priorities that government stakeholders want to exhibit in the governance process. 


\section{Discussion}

The use of MCDA, Delphi studies, and other analytic tools are geared toward improving the identification of policy options and decisionmaking under situations of high complexity and uncertainty, as in the investigated case of cyberplasm. As the technology itself is further researched and developed, such decision analytic tools can help to focus investigations on oversight and policy choices "upstream" from final technological development and use (Kuzma et al. 2008; Linkov et al. 2013). Decisions about how best to prepare for governing emerging technologies such as cyberplasm have often made use of multiple lines of quantitative and qualitative information (such as modeling and monitoring, risk analysis, cost-benefit analysis, and stakeholder preferences), which are typically combined in a subjective and unstructured manner (Linkov et al. 2006). This ad hoc decision basis may ignore crucial factors for governance program success and is likely to yield a suboptimal and regretted allocation of resources. Regulators, in partnership with stakeholders, can significantly benefit from new modes of decision analysis like the one presented here that allow them to acquire and analyze information about emerging technological applications in a manner that systematically incorporates qualitative and quantitative information regarding expert-derived risk and benefit data, along with defined governance options. Tools such as the one described in this paper can also be integrated into stakeholder and public participation exercises to develop scenarios and help frame management options under conditions of high uncertainty and ambiguity (IRGC 2006, 2015).

Of the six policy options presented for the case of cyberplasm, the model calculated ERB pre-research is the most optimal governance option. This form of governance is characterized by the need for approval from an external government review board prior to conducting research. It supports the IRGC recommendation for wider participatory approaches (i.e. ERB) under areas of high uncertainty and ambiguity that include confinement and the stepwise introduction of emerging technologies into society (IRGC 2006). This conclusion was based on expert panel considerations of the potential hazards associated with the development of the technology, as well as its potential applications. This governance option demonstrated only slight net performance optimality compared with other governance options, including secured laboratory study, IRB pre-research, and ERB post-research, respectively. Notably, the recommended governance option calculated by the MCDA system can be receptive to ranked weightings of its various criteria (social, risks, political, and economic). However, inaccurate weighting of criteria may unjustly skew calculations. A priori ranking of criteria importance must be considered carefully prior to calculation of recommended decision outputs and should reflect current environment and legislation schemes under which the technology being assessed will be governed. Criteria ranking could also be performed in wider deliberative approaches to risk governance whereby stakeholders, interested and affected parties, and experts convene to explore risk management options and scenarios (IRGC 2015). Only then can these tools be used appropriately to guide decisionmaking for cases such as cyberplasm that are characterized by high complexity and uncertainty, in order to highlight oversight and policy choices "upstream" from final technological development and use (Kuzma et al. 2008; Linkov et al. 2013).

Synthetic biology decisionmakers, stakeholders, and the public are likely to benefit from transparent, analytic approaches that identify and prioritize proactive governance options based on granular needs-assessments that incorporate expert-derived data and confidence estimates. As risk and benefit data analysis for emerging technologies continue to improve, analytic schema like the one portrayed here for cyberplasm can be updated and evolved to incorporate new criteria or extinguish irrelevant factors from the analysis. Furthermore, this investigation of policy options for the future governance of cyberplasm is just one example of the benefit of MCDA as an option to inform initiatives of early-stage governance of technologies while they are still in pre-production. SB technologies, nanotechnologies, and other revolutionary technological applications are being developed at a frenetic pace. However, there is a dearth of proactive rather than reactive initiatives being undertaken to evolve governance structures. Forecasting policy options of emerging technologies will likely require greater governance involvement in early-stages of development because of the revolutionary nature of technologies like cyberplasm. Identifying and preparing for the use of hybrid and converging technologies increases the uncertainty of the potential impacts, and even uses of such technologies. In such cases of extreme uncertainty, the use of real data from expert studies like this one seems to be a most practical benchmark from which we can forecast governance needs prior to technological diffusion.

Overall, while this paper uses cyberplasm as a "test case" for evaluating SB risks, benefits, and governance options, it could easily be applied to a whole host of emerging technology applications that lack objective data yet have clear and emerging governance needs to be met. There is no definitive guide to carrying out such research, but in general, using a 
transparent and robust interview and survey methodology while systematically selecting experts for inclusion would offer helpful and much needed early stage policy and governance advice. This study also notes that governance decisions are likely to be influenced by the prioritized needs of governing bodies and stakeholders. For instance, should a governing body or stakeholders prioritize economic outcomes over others, the MCDA rubric and the DECERNS system provide calibrated flexibility to assist with such needs and prescribe alternate policy options that can be calibrated to reflect such preferences and/or adopt new information about the technology's risk/benefit trade-offs related to economics.

While we argue that this approach is a promising step forward in anticipatory governance and that tools like the one demonstrated here are likely to remain among the best viable options for guiding early-stage governance of technologies, certain limitations should be noted. Specifically, using the Delphi method, highly influential group members can have an effect during the face-to-face workshop (Franklin \& Hart 2007). To limit such effects, the Delphi study used here included multiple rounds of interviews, in which each respondent was interviewed before and after workshop participation. Future work of this type may consider oversampling based on expected attrition, and may modify their use of the Delphi method to better reflect their programmatic aims (Linstone \& Turoff 2002).

\section{Conclusion}

While the decision analytic system described in this paper does not inherently advocate any particular policy options, and where its outputs are likely to change as the field continues to develop and expand into commercialization, this sort of investigation may serve as a heuristic to decisionmaking that prioritizes governance needs based on stakeholder and expert elicitation. Ideally, future inputs of the tool would be revised and ranked based on wider deliberation of experts, stakeholders, and policymakers, and outputs of the tool would be integrated into participatory processes to construct different governance scenarios and risk management options.

\section{Acknowledgments}

The study reported here is situated within a larger grant project funded by the Alfred P. Sloan Foundation grant (\#G2013-3-02) "Looking Forward to Synthetic Biology Governance: Convergent Research Cases to Promote Policymaking and Dialogue." The authors gratefully acknowledge the support of the Genetic Engineering and Society Center at North Carolina State University and Sharon Stauffer, Christina Ndoh, and Sheron King for their help with the Sloan study data collection. Finally, the authors would like to acknowledge Dr. Boris Yatsalo for making the DECERNS software available for this paper.

This work was funded in part by the US Army Environmental Quality Research Program (63372803E00). Permission was granted by the authority of the Chief of Engineers to publish this information.

\section{References}

Bates ME, Grieger KD, Trump BD, Keisler JM, Plourde KJ, Linkov I (2016) Emerging Technologies for Environmental Remediation: Integrating Data and Judgment. Environmental Science \& Technology 50, 349-358.

Belton V (1986) A Comparison of the Analytic Hierarchy Process and a Simple Multi-attribute Value Function. European Journal of Operational Research 26, 7-21.

Berube D, Cummings C, Cacciatore M, Scheufele D, Kalin J (2011) Characteristics and Classification of Nanoparticles: Expert Delphi Survey. Nanotoxicology 5, 236-243.

Calvert J, Martin P (2009) The Role of Social Scientists in Synthetic Biology. EMBO Reports, 10, 201-204.

Church GM, Elowitz MB, Smolke CD, Voigt CA, Weiss R (2014) Realizing the Potential of Synthetic Biology. Nature Reviews Molecular Cell Biology 15, 289-294.

deLorenzo V (2010) Environmental Biosafety in the Age of Synthetic Biology: Do We Really Need a Radical New Approach? BioEssays 32, 926-931.

Franklin KK, Hart JK (2007) Idea generation and exploration: benefits and limitations of the policy Delphi research method. Innovative Higher Education 31, 237-246.

Gibson DG, Glass JI, Lartigue C, Noskov VN, Chuang RY, Algire MA et al. (2010) Creation of a Bacterial Cell Controlled by a Chemically Synthesized Genome. Science 329, 52-56. 
Grubišić V, Parpura V (2015) Diversity in the Utilization of Glucose and Lactate in Synthetic Mammalian Myotubes Generated by Engineered Configurations of MyoD and E12 in Otherwise Non-differentiation Growth Conditions. Biomaterials, 43, 50-60.

Hutchison CA III, Chuang RY, Noskov VN, Assad-Garcia N, Deerinck TJ, Ellisman MH et al. (2016) Design and Synthesis of a Minimal Bacterial Genome. Science 351, 1414-U73.

IRGC (International Risk Governance Council) (2006) Risk Governance: Toward an Integrative Approach. IRGC, Geneva. [Last accessed 14 November 2016] Available from URL: https://www.irgc.org/IMG/pdf/IRGC_WP_No_1_Risk_Governance_ reprinted_version_.pdf

IRGC (International Risk Governance Councile) (2015). IRGC Guidelines for Emerging Risk Governance. IRGC, Geneva. [Last accessed 1 April 2016] Available from URL: http://www.mmc.com/content/dam/mmc-web/Global-Risk-Center/Files/IRGCEmerging-Risk-WEB-31Mar.pdf.

Kuzma J, Najmaie P, Larson J (2009) Evaluating Oversight Systems for Emerging Technologies: A Case Study of Genetically Engineered Organisms. Journal of Law Medicine \& Ethics 37, 546-586.

Kuzma J, Paradise J, Ramachandran G, Kim J, Kokotovich A, Wolf SM (2008) An Integrated Approach to Oversight Assessment for Emerging Technologies. Risk Analysis 28, 1197-1220.

Kuzma J, Tanji T (2010) Unpackaging Synthetic Biology: Identification of Oversight Policy Problems and Options. Regulation \& Governance 4, 92-112.

Linkov I, Bates ME, Trump BD, Seager TP, Chappell MA, Keisler JM (2013) For Nanotechnology Decisions, Use Decision Analysis. Nano Today 8, 5-10.

Linkov I, Moberg E (2011) Multi-criteria Decision Analysis: Environmental Applications and Case Studies. CRC Press, Boca Raton, FL. Linkov I, Rosoff H, Valverde LJ, Bates ME, Trump B, Friedman D et al. (2012) Civilian Response Corps Force Review: The Application of Multi-criteria Decision Analysis to Prioritize Skills Required for Future Diplomatic Missions. Journal of Multi-Criteria Decision Analysis 19, 155-168.

Linkov I, Satterstrom FK, Kiker G, Batchelor C, Bridges T, Ferguson E (2006) From Comparative Risk Assessment to Multi-criteria Decision Analysis and Adaptive Management: Recent Developments and Applications. Environment International 32, $1072-1093$.

Linstone HA, Turoff M (eds) (2002) The Delphi Method: Techniques and Applications. Addison-Wesley Publishing Company Inc, Reading, M.A.

Mohan M, Trump BD, Bates ME, Monica JC Jr, Linkov I (2012) Integrating Legal Liabilities in Nanomanufacturing Risk Management. Environmental Science \& Technology 46, 7955-7962.

Patton MQ (2005) Qualitative Research. Encyclopedia of Statistics in Behavioral Science. John Wiley \& Sons Ltd., New York, NY.

Saltelli A, Tarantola S, Chan K (1999) A Role for Sensitivity Analysis in Presenting the Results from MCDA Studies to Decision Makers. Journal of Multi-Criteria Decision Analysis 8, 139-145.

Stewart TJ (2005) Dealing with uncertainties in MCDA. In: Figueira J. et al. (eds) Multiple Criteria Decision Analysis: State of the Art Surveys, pp. 445-466. Springer, New York.

Subramanian V, Semenzin E, Hristozov D, Zabeo A, Malsch I, McAlea E et al. (2016) Sustainable Nanotechnology Decision Support System: Bridging Risk Management, Sustainable Innovation and Risk Governance. Journal of Nanoparticle Research 18 .Article 89

Szondy D (2012) “Interface Scaffolds" Could Wire Prosthetics Directly into Amputees' Nervous Systems. New Atlas 5 Mar 2012. [Last accessed 1 September 2016] Available from URL: http://www.gizmag.com/nerve-prostheses-interface-scaffolds/21646/

Tucker JB, Zilinskas RA (2006) The Promise and Perils of Synthetic Biology. New Atlantis, 12, 25-45.

Voigt CA (2012) Synthetic Biology: Bacteria Collaborate to Sense Arsenic. Nature 481, 33-34.

Woodrow Wilson Center (2016) Cyberplasm. Synthetic Biology Project. [Accessed 14 November 2016] Available from URL: http:// www.synbioproject.org/cpi/applications/cyberplasm/.

Yatsalo B, Didenko V, Gritsyuk S, Sullivan T (2015) Decerns: A Framework for Multi-criteria Decision Analysis. International Journal of Computational Intelligence Systems 8, 467-489.

Yatsalo B, Kiker G, Kim J, Bridges T, Seager T, Gardner K et al. (2007) Application of Multicriteria Decision Analysis Tools to Two Contaminated Sediment Case Studies. Integrated Environmental Assessment and Management, 3, 223-233. 